\title{
EFFECT OF A SIDE COLLISION-AVOIDANCE SIGNAL ON SIMULATED DRIVING WITH A NAVIGATION SYSTEM
}

\author{
Dong-Yuan Debbie Wang \\ University of North Florida, Jacksonville, Florida USA \\ E-mail: dwang@unf.edu \\ David F. Pick \\ Purdue University Calumet, Hammond, Indiana USA \\ E-mail: pick@calumet.purdue.edu \\ Robert W. Proctor \\ Purdue University, West Lafayette, Indiana USA \\ E-mail: proctor@psych.purdue.edu \\ Yi (Derek) Ye \\ University of North Florida, Jacksonville, Florida USA \\ E-mail: yye@unf.edu
}

\begin{abstract}
Summary: Effects of a side collision-avoidance system (SCAS) signal on driving behavior were examined in an environment in which a nagivation signal was also used. Sixteen undergraduate students participated in this study, and a computerbased STISIM driving simulator was used in the project. Subjects were asked to respond to two signals, a visually displayed directional signal generated by a simulated navigation system (NAS) and a monaural auditory tone from a simulated SCAS presented after a NAS signal. Subjects were instructed that the SCAS signal conveyed directional information about an impending threat (the location of the danger from which they were to turn, or the escape direction toward which they were to turn). Contrary to previous findings in a non-driving environment (Wang et al., 2003), response time (RT) was significantly shorter for the group in which the location of the SCAS signal was spatially compatible with the location of the danger than for the group in which the SCAS signal location was incompatible with the location of the danger. Mean RT was not significantly shorter when the direction of the NAS signal and the location of the SCAS signal corresponded than when they did not. Given that subjects tended to withhold responding until they perceived the encroaching car, the benefit of a SCAS may be to direct a driver's attention in the direction of an impending threat before the driver would ordinarily detect it.
\end{abstract}

\section{INTRODUCTION}

Motor vehicle accidents have become one of the top causes of death in the U.S. With advances in technology, increasing numbers of intelligent systems are being built into vehicles to assist driving and to improve safety and efficiency. Navigation systems (NAS) have become an option for most vehicles in 2007 models. These systems provide guidance when driving in an unfamiliar area, as well as efficient routes that save time and fuel. They also provide the indirect potential safety benefit of reducing uncertainty and decreasing the possibility of a driver making a lastminute turn. Other intelligent systems, which are intentionally designed to reduce fatalities and injury, include collision avoidance systems. A collision avoidance system uses sensors to provide warnings of impending collisions. Qualitative research about consumer responses to automotive 
crash-avoidance devices indicated that "most of the participants thought that this product concept was a good idea, and they expressed interest in having the option on their next new vehicle" (Charles River Associates, 1998).

Current collision avoidance systems were not designed to prevent all types of collisions but to prevent a specific type of collision (Dingus, Jahns, Horowitz, \& Knipling, 1998). The collision avoidance systems for backing up and blind spot detection were the two most needed (Charles River Associates, 1998). Effective collision avoidance systems are available for prevention of rear-end crashes, and effective side collision avoidance systems (SCAS) are expected to be available soon. The development of SCASs for side crashes has been complicated by the fact that vehicles in adjacent lanes can be very close to each other without presenting an imminent danger, and normal lane-change behavior produces occasional instances of high closure rates.

Previous research indicates that directional alerts of potential threats are preferred and necessary for a SCAS (Campbell et al., 1996; Hancock \& Parasuraman, 1992). An important concern in the design of a SCAS is how to best present a warning signal to convey information to drivers that will induce fast and accurate responses. Because SCAS alerts are directional, their relationship with the appropriate response may be spatially compatible or incompatible. Low display-control compatibility may confuse the driver and prolong response time (RT) to a threat (Campbell et al., 1996). Wang et al. (2003) conducted an experiment with a steering wheel to test the effect of the location of an auditory warning signal on RT and error rate when simulated hazards occurred on the right or left. RT was shorter for the subjects who were told that the SCAS signal indicated the evasive direction than for those who were told that the tone indicated the location of the hazard. That is, the results showed a standard spatial compatibility effect where it was easier to respond in the direction of the signal rather than away from it.

An issue that has not been considered is how to integrate all in-vehicle systems in a safe and effective human-centered design. Displays and controls should be studied in an integrated environment, especially when more than one directional signal can be presented successively or concurrently. Responses to a directional signal from one component system may be affected by an irrelevant directional cue from another component system, and benefits of compatibility when tested in isolation may be lost when tasks of responding to different intelligent systems are mixed (Proctor \& Vu, 2006). For example, when a navigation signal presents visual information (alone or with auditory advice) just prior to, or coincident with, a collision warning signal, the navigation signal provides location information that is irrelevant with regard to the warning signal, and its spatial correspondence with the warning may indeed influence response to the warning signal. Such correspondence effects of irrelevant spatial stimulus information on performance have been obtained in many studies (Hommel \& Prinz, 1997; Wang, et al., 2003). How a directional NAS signal might affect responses to the SCAS signal has not been tested. In fact, the issue of how to make the two systems compatible when operated together in an automobile has not been addressed.

In this study the effects of warning signals on the evasive behavior in collision scenarios were investigated using a driving simulator. We were interested in two questions: First, should a directional warning signal for impending collision indicate the location of the danger or the escape direction? Second, does a NAS signal influence a response to a SCAS signal? 


\section{METHOD}

\section{Subjects}

Sixteen undergraduate students from the University of North Florida participated to earn research credit. Eight students were assigned to each of two groups.

\section{Apparatus and Stimuli}

A STISIM-Model 100 driving simulator was used in the project. The STISIM is a PC-based simulator with an interactive Logitech steering wheel, brake pedal, and accelerator. The driving scene is displayed on a 17' Dell monitor located in front of the subject. In this study, the roadway was designed as an urban road with 6 lanes (three lanes in each direction), and the speed limit was $30 \mathrm{miles} / \mathrm{hr}$. Subjects were asked to follow the traffic signs and drive in the center lane. In the driving simulation, cars continuously approached the driver's vehicle in the opposite lanes of the road. There was relatively less traffic on the driver's side of the road, except for a few cars that passed the driver's vehicle.

Two signals were used. One was a visual NAS signal consisting of three solid arrows presented successively on the left- or right-bottom corner. This method resulted in apparent movement of the arrow from left to right or right to left. The SCAS signal was presented $0.5 \mathrm{~s}$ before the appearance of a threatening vehicle. The SCAS signal was a 1,100-Hz monaural tone presented to the left or right ear at $80 \mathrm{~dB}$ for $500 \mathrm{~ms}$ through Panasonic stereo headphones (RP-HT355).

\section{Procedure}

The subjects, all licensed drivers, were interviewed briefly before being allowed to operate the driving simulator, following a procedure adapted from Ranney et al. (2002). Three potential subjects were turned away due to a prior accident, diabetes, and a possible pregnancy. After the interview, the eligible drivers read and signed an informed consent form.

A 10-min training session was conducted to allow subjects to become accustomed to controlling the simulated vehicle and familiarized with the NAS and SCAS. After brief instructions, they performed a scenario, with the assistance of the experimenter, which lasted about 8 minutes. Subjects were instructed to respond to the NAS signal by pressing the left- or right-turn button when they saw the next intersection. Subjects in one group were told that the SCAS tone indicated the location of the danger from which they were to turn away: the tone location in this group was spatially compatible with the location of the danger but incompatible with the escape direction. Subjects in this group were instructed to avoid a collision by braking and turning the wheel to merge into the other lanes as they saw fit.

The other group was instructed that the warning tone indicated the escape direction toward which they were to turn: the SCAS tone location in this group was compatible with the escape direction but incompatible with the location of the danger. The experimenter explained that the correct response was to brake and turn away from a dangerous car that was passing and merging into their lane. The subject was told that it was important that s/he control the vehicle, and that they 
were to be the final judge of when or whether to respond to any signal during the virtual drive. It was emphasized that safety was their primary concern, even though the environment was simulated.

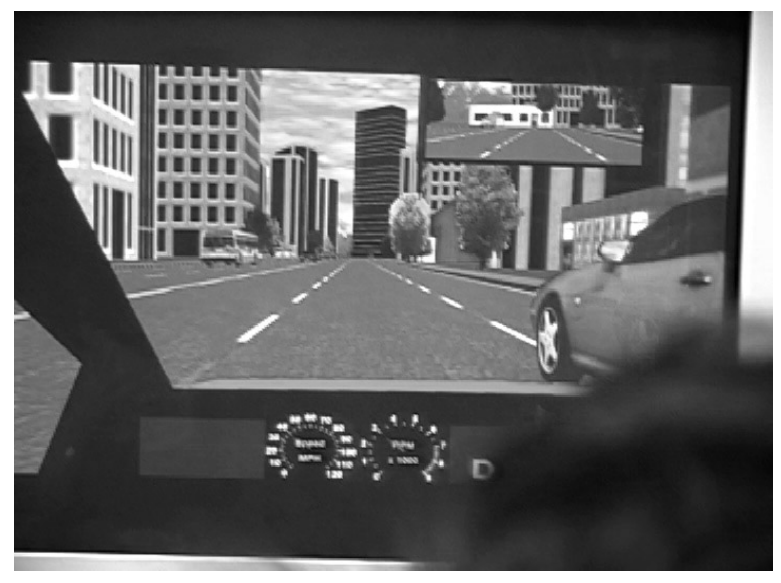

Figure 1. A car merging to the center lane

Subjects participated in a 40-min test session which included two sub-sessions, each of which contained presentation of 12 NAS signals, eight of which were followed by a SCAS signal. Subjects were allowed a short break between sub-sessions. During each sub-session, elapsed time since the beginning of the run (in seconds) was recorded. Each SCAS signal was presented $3.0 \mathrm{~s}$ after onset of a NAS signal and $0.5 \mathrm{~s}$ before the appearance of an encroaching vehicle, which merged into the center lane occupied by the driver's simulated vehicle after another $0.5 \mathrm{~s}$ (see Figure 1). The driver's longitudinal velocity (in $\mathrm{ft} / \mathrm{s}$ ) and longitudinal acceleration (in $\mathrm{ft} / \mathrm{s}^{2}$ ) were recorded for $9 \mathrm{~s}$ beginning with the onset of every SCAS signal.

\section{RESULTS}

We were interested in two questions: how to present a directional warning signal for collision avoidance (indicating the location of the danger or the escape direction) and how the NAS signal would influence responses to the SCAS signal. The two questions were answered by testing two effects on RT to the potential danger: (a) the compatibility effect of the SCAS signal with the location of the danger, and (b) the correspondence effect of the NAS signal and the SCAS signal on the response to the potential danger.

In a pilot experiment, we noted that drivers did not respond initially to the SCAS warning by turning the wheel, but rather only pressed the brake gently. They braked more forcefully and turned the wheel only when they saw the encroaching vehicle. Acceleration change is directly linked to force change on the brake. To obtain RT to the SCAS signal, we plotted elapsed time since the onset of the SCAS signal vs. the driver's longitudinal acceleration (in $\mathrm{ft} / \mathrm{s}^{2}$ ) and determined when a response was made by calculating the first-order derivative after $1 \mathrm{~s}$ of signal presentation and locating the initial point of deceleration. A typical relationship between elapsed time and longitudinal acceleration is displayed in Figure 2, for which the RT was measured as $4.49 \mathrm{~s}$. If a curve varied unsystematically, RT could not be determined and the trial was not 
included in any analysis. In addition, trials with RTs that were too short (anticipations $<1 \mathrm{~s}$ ) or too long (RT $>7 \mathrm{~s}$ ) were not included. If a crash occurred during a trial, no RT was recorded, but the program resumed at a point prior to the crash, with the car in the center lane, and the scenario was replayed. These criteria resulted in a total of 116 trials (63 in the group turning toward the SCAS and 53 in the group turning away from it) being included in the RT analysis.

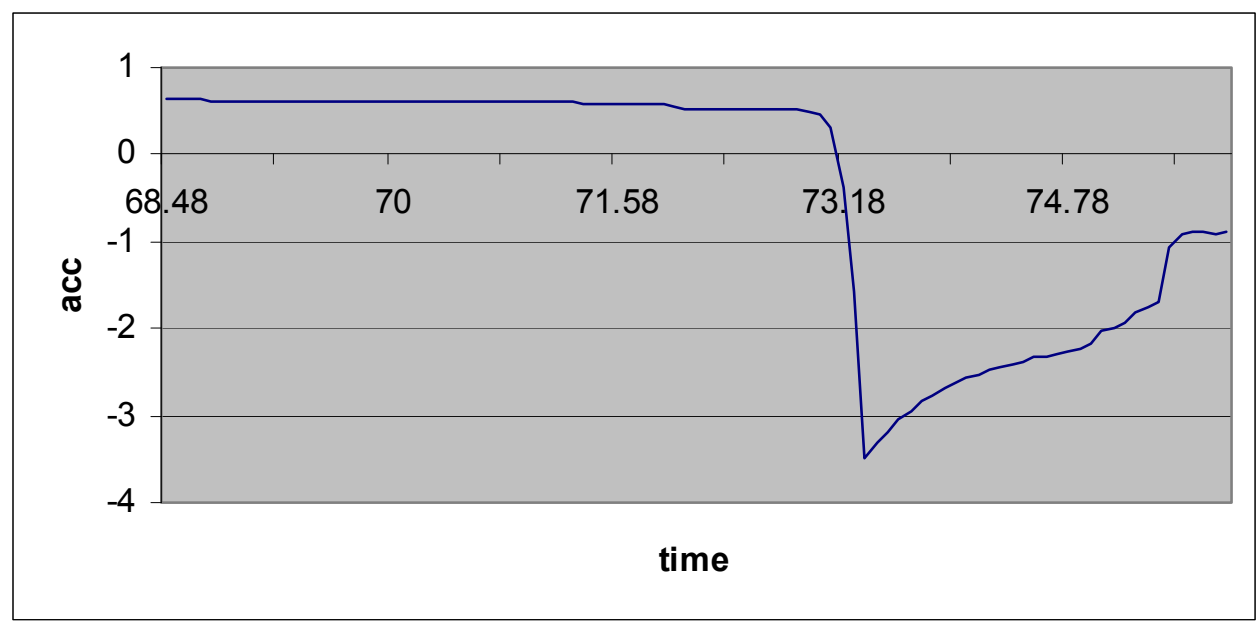

Figure 2. Longitudinal acceleration changes as a function of elapsed time

Because some individual subjects contributed only a few data points, the data were analyzed by trials as opposed to by subjects. RT for the group in which the SCAS signaled the location of the danger $(M=3.86 \mathrm{~s})$ was significantly shorter than RT for the group in which the SCAS signaled the safe avoidance direction $(M=4.33 \mathrm{~s}), F(1,108)=3.95, p<.05$. Mean RT was shorter when the direction of the NAS signal and the tone location of the SCAS signal corresponded than when they did not $(M \mathrm{~s}=3.89 \mathrm{~s}$ and $4.29 \mathrm{~s})$, but this difference was not significant, $F(1,108)<1$. Thus, the results showed shorter RT to the SCAS signal when the appropriate response was to turn away from it than toward it, but correspondence of the NAS signal with the SCAS signal had only a nonsignificant effect.

\section{DISCUSSION}

Standard spatial compatibility effects lead to the expectation that it would be most beneficial to have a warning tone correspond to the desired response direction. Previous results showed that RT to an auditory signal in a non-driving environment was shorter when the signal represented the escape direction rather than the direction of the hazard (Wang et al., 2003). However, the current study conducted in a simulated driving environment showed the opposite relation. The present results can be reconciled with the previous findings as follows: first, in the prior study, subjects responded immediately to the auditory signal, whereas in the present study they often waited until the appearance of the intruding vehicle before responding; second, the failure to obtain a significant correspondence effect between the irrelevant location information presented by the NAS signal and the relevant location information of the SCAS signal could be due to noise in the data obscuring an effect. However, it also could be a consequence of a lack of trust in the SCAS system, as suggested by the delay in responding to the warning. If the latter is the 
case, if trust in the system can be established through experience, drivers may directly respond to a SCAS signal and show a correspondence effect with the NAS.

The current study demonstrates that under more realistic conditions than those used by Wang et al. (2003), drivers did not respond to the SCAS signal immediately. Rather, they withheld their response until they perceived the encroaching car. Thus, the benefit of a SCAS may be to direct the driver's attention in the direction of an impending threat before the driver would ordinarily detect it. This benefit of attending to the location of the impending danger is consistent with studies showing that cuing attention to a visual location results in facilitation in processing the information at that location (McDonald et al., 2000).

\section{REFERENCES}

Campbell, J.L., Hooey, B.L, Camey, C., Hanowski, R.J., Gore, B.F., Kantowitz, B.H., \& Mitchell, E. (1996). Investigation of alternative displays for side collision avoidance systems. (Technical report). US Department of Transportation: National Highway Traffic Safety Administration.

Charles River Associates, Inc. (1998, April). Consumer acceptance of automotive crash avoidance devices (CRA Project NO. 852-05). Boston, MA: Author, 32.

Dingus, T.A., Jahns, S.K., Horowitz, A.D., \& Knipling, R. (1998). Human factors design issues for crash avoidance systems. In W. Barfield \& T. A. Dingus. (Eds.), Human factors in intelligent transportation systems. Mahwah, NJ: Erlbaum, 55-94.

Hancock, P.A., \& Parasuraman, R. (1992). Human factors and safety in the design of intelligent vehicle-highway systems (IVHS). Journal of Safety Research, 23, 181-198.

Hommel, B., \& Prinz, W. (Eds.) (1997). Theoretical issues in stimulus-response compatibility. Amsterdam: North-Holland.

McDonald, J.J., Teder-Saelejaervi, W.A., \& Hillyard, S.A. (2000). Involuntary orienting to sound improves visual perception. Nature, 407, 906-908.

Proctor, R.W., \& Vu, K.-P.L. (2006). Stimulus-response compatibility principles: Data, theory, and application. Boca Raton, FL: CRC Press.

Ranney, T.A., Heydinger, G., Watson, G., Salaani, K., Mazzae, E.N., \& Grygier, P. (2002). Invetigation of driver reactions to tread separation scenarios in the national advanced driving simulator (NADS). (Technical report). US Department of Transportation: National Highway Traffic Safety Administration.

Wang, D-Y., Y., Proctor, R.W., \& Pick, D.F. (2003). Stimulus-response compatibility effects for warning signals and steering responses. Proceedings of the 2 nd International Driving Symposium on Human factors in Driver Assessment, Training and Vehicle Design. Park City, UT. 